\title{
Finite-time Chaos Synchronization Control of Permanent Magnet Synchronous Motor with Nonuniform Air Gap based on Load Sliding Mode Observation
}

\author{
Chuansheng Tang ${ }^{1 *}$, Jie Yang ${ }^{2}$, Qiang Zhao ${ }^{3}$, Xuandong $\mathrm{Wu}^{3}$ and $\mathrm{Tao}^{\mathrm{Li}}{ }^{4}$ \\ ${ }^{1}$ Nanyang Institute of Technology, Nanyang Henan 473004, China \\ ${ }^{2}$ Henan Institute of Technology, Xinxiang Henan 453000, China \\ ${ }^{3}$ Wolong Electric Nanyang explosion proof Group Co., Ltd, Nanyang Henan 473004, China \\ ${ }^{4}$ Department of Informatics, University of Zurich, Zurich 8050, Switzerland
}

Received 4 January 2020; Accepted 26 March 2020

\begin{abstract}
Permanent magnet synchronous motor (PMSM) has a simple structure, small volume, high efficiency, and high power factor. However, their motor parameters are affected by strong coupling and nonlinear factors, such as current, flux, and speed, all of which can lead to the chaos phenomenon. A finite-time chaos synchronization method based on a sliding mode observer was proposed in this work to improve the effects of parameter uncertainty and load disturbance on the chaotic system performance of PMSM with a nonuniform air gap and the dynamic performance of the chaos synchronization system. First, the uncertain load was observed online by the sliding mode observer. Second, the controller of the PMSM system was designed on basis of the theory of sliding mode and theory of finite time stability. Finally, the effectiveness of the proposed method was verified by simulation. Results demonstrate that the designed sliding mode observer can realize the precise tracking of sudden loads quickly and steadily within $2 s$, the proposed method within $2.5 \mathrm{~s}$ has a faster response than adaptive control about $20 \mathrm{~s}$ and finite-time synchronous control about $13 \mathrm{~s}$ under the same conditions. Moreover, the synchronization accuracy of the proposed method is not affected when the parameters change, whereas that of adaptive synchronization control is about $13 \mathrm{~s}$. The proposed method exhibits high chaos synchronization tracking performance and strongly suppressed the external disturbance of the system. The proposed method thus provides a good prospect for improving the theory of motor synchronous control and its application to engineering practice.
\end{abstract}

Keywords: Nonuniform air gap, PMSM, Chaos synchronization, Sliding mode observer, Finite time

\section{Introduction}

Permanent magnet synchronous motor (PMSM) has a simple structure, small size, high efficiency, and low energy consumption. Hence, PMSM is widely used in the fields of instruments, aerospace, $\mathrm{CNC}$ machine tools, and medical devices. In recent years, extensive study has explored the stability and reliability of PMSM. Since the discovery of chaos in motor drive systems in the 1990s, its analysis and control have elicited increasing attention. Chaos is common in induction motor, PMSM, DC motor, switched reluctance motors, and other servo systems [1].

Early study on chaos in motor drives mainly focused on the identification of chaos [2]. At the beginning, the boundary of chaos in a motor drive is often mistaken as noise when it is small and as an unstable operation of the motor system when it is large. However, chaos is different from unsteadiness phenomena, such as out of step in motor drives. The chaos in a motor is a complex steady-state behavior with the following characteristics. As the motor parameters change, the system presents a violent oscillation due to speed or torque, the unsteadiness of the control performance, and the irregular electromagnetic noise of the system. This oscillation seriously affects the dynamic performance of the system. The change of motor parameters and the disturbance of external load greatly increase the

*E-mail address: tcs111@163.com ISSN: 1791-2377 @ 2020 School of Science, IHU. All rights reserved. doi:10.25103/iestr.132.10 complexity of control systems; hence, chaos synchronization control with parameter uncertainty has been studied $[3,4]$. However, the influence of external disturbance on control systems has not been extensively investigated, with control accuracy being the main focus. Moreover, the study on the dynamic performance of control systems is limited. Therefore, the control of the adverse effects of external disturbance has become a study hot spot. Meanwhile, the chaos behavior of motors is beneficial in certain circumstances. For example, the chaos phenomenon of a motor is employed to improve the efficiency of grinding and mixing, which is an issue in chaos anticontrol and in the synchronous control of motors [5].

To address the problem of the synchronous control of PMSM systems with external disturbance and uncertain system parameters, this study designs a controller for PMSM systems for chaos synchronization on the basis of sliding mode theory and finite-time stability theory. The proposed controller can enhance the robustness, response speed, and stability of PMSM systems precisely. The results of this work provide a reference for the improvement of motors' chaos synchronization theory and its practical application.

\section{State of the art}

Many chaos synchronization control methods are currently available, but only a few are meant for PMSM, such as 
Chuansheng Tang, Jie Yang, Qiang Zhao, Xuandong Wu and Tao Li/

Journal of Engineering Science and Technology Review 13 (2) (2020) 63 - 71

feedback synchronization [6,7], fuzzy proportional-integralderivative (PID) synchronization [8], adaptive synchronization [9-11], sliding mode synchronization $[12,13]$, pole assignment synchronization [14], active synchronization [15], reverse synchronization [16,17], time delay synchronization [18], and fuzzy synchronization $[19,20]$. Yin et al. first analyzed the dynamic characteristics of chaos in a typical multiscroll Chua system and a brushless DC motor (BLDCM) system [6]. Based on the Lyapunov stability principle, a nonlinear feedback synchronous controller was designed for the two systems to realize the effective control of the BLDCM. MATLAB software was employed to simulate the control of the whole system, and the results verified the correctness and validity of the synchronous control method. However, the designed controller does not consider the influence of system parameter change on system performance. Torres et al. proposed a master-slave synchronization method on the basis of induction motor drives [7]. The proposed method adopted the input torque of each joint of the robot as the reference torque of the induction motor and the feedback control law to realize the synchronous position tracking of the desired trajectory. Lyapunov analysis showed that the controller achieved the semiglobal exponential convergence of synchronous closed-loop errors, and the simulation results verified the validity of the proposed method. The controller designed with the above method depends on the mathematical model of the system. When the system parameters change, its performance declines, or it even fails. Ranjbar et al. combined fuzzy control with PID control and adjusted the gain of the PID controller online through a fuzzy control rule to realize the antidisturbance ability of the system model parameters, finally overcome the influence of parameter changes on the synchronous control performance of the system [8]. However, the controller still depends on the mathematical model of the system, the fuzzy control rules and structure are difficult to determine, a large number of rules could increase the response time and reduce the corresponding ability, and a small number of rules could not resist disturbances. Kim et al. proposed a simple adaptive synchronization method for the chaotic system of a PMSM with uncertain parameters and analyzed the stable convergence of the closed-loop system response using Lyapunov theory [9]. The proposed method does not need all the parameters of the PMSM and could successfully achieve uncertainty for the chaos synchronization of the PMSM to provide an effective method when model parameters change. To improve the performance of control systems, the study adopted adaptive synchronization by introducing an adaptive mechanism to the online estimation of system parameters. However, the introduction of the adaptive mechanism inevitably increases the cost of the system and reduces the response capability of the system to a certain extent. Zhang et al. proposed and implemented a new speed synchronization control method for multiinduction motors under uncertain chaotic systems [10]. The proposed method is a nonlinear control method that achieves adaptive time-delay feedback control by adjusting the reference torque of the direct torque control device on the basis of the stator flux adjustment. With the proposed control method, the multi-induction motor could realize synchronous chaotic speeds within a controllable boundary, and adaptive control showed strong robustness to changes in motor parameters. However, the introduction of the adaptive mechanism increased the complexity of the controller structure and the dynamic regulation time of the system to a certain extent. For a PMSM with a nonlinear structure, Liu et al. proposed a parameter identification method on the basis of adaptive synchronization. During identification, the dynamic response of the PMSM is synchronized with another system with a similar dynamic structure [11]. The algorithm uses globally convergent feedback control for the reference model. The simulation and experiment on the parameter identification of the PMSM servo system were carried out to verify the proposed identification method, and the results showed that the proposed method is effective. Yang et al. took the chaotic motion of a PMSM as the study object and proposed a chaos synchronization control method with a sliding mode variable structure for PMSM with disturbance [12]. The proposed method is a self-adaptive controller with strong robustness and can realize sliding mode even in the case of external disturbance and parameter uncertainty. The numerical simulation results showed that the proposed method could be applied to PMSM. Although the synchronous control of chaotic motion was satisfactory, the sliding mode synchronous control method needs to meet certain matching conditions, and the system suffers from inherent chattering. On the basis of active control, Yu et al. took a doubly fed wind turbine as the study object and designed an active sliding mode controller, which can ensure system stability under any initial condition [13]. The proposed method can realize the structure of the linear and nonlinear terms of a system and effectively simplify the controller structure. However, it cannot eliminate the inherent chattering phenomenon of sliding mode control. Zaher first analyzed the dynamic characteristics of PMSM and designed a synchronous state observer using only angular velocity as feedback on the basis of the similarity between the mathematical model of a PMSM and the famous chaotic Lorentz system [14]. The simulation results verified the effectiveness of the controller in eliminating chaotic oscillation under a single feedback signal, and the advantages of the controller were further verified by comparison with the conventional PID controller. Vafaei et al. studied the chaotic behavior of a fractional-order PMSM system and designed an active synchronous control method on the basis of the stability theory of a fractional-order PMSM system [15]. The proposed method is simple and flexible and is thus suitable for design and practical applications. The simulation results showed that the proposed method possesses good control effect and robustness for fractional-order PMSM systems. However, the study only considered parameter change and focused on the steady-state performance of PMSM systems, hence the difficulty in making the proposed method meet the requirements of load disturbance and dynamic performance, including adjustment time. Wang et al. proposed a new composite PMSM system by introducing the concept of complex current and reset cross-coupling term and analyzed its performance [16]. On the basis of a complex PMSM system, the backstepping method was employed to design the controller, which realizes the lag synchronization of real and virtual parts. The numerical simulation results verified the effectiveness of the controller, and the design of the controller depends on the model parameters of the system, and the derivation of the system state could continue in the backstepping design, resulting in calculation explosion. Yang et al. used the backstepping synchronization control principle to make a controlled chaotic system degenerate gradually into a stable system [17]. According to the Lyapunov stability principle, the designed controller could make two chaotic systems with the same structure but 
Chuansheng Tang, Jie Yang, Qiang Zhao, Xuandong Wu and Tao Li/

Journal of Engineering Science and Technology Review 13 (2) (2020) 63 - 71

different parameters gradually reach synchronization, but it could not eliminate the computational explosion phenomenon caused by the backstepping control, and it could not restrain the changes in system parameters. Thus, the proposed method must be combined with adaptive control or sliding mode control. Li et al. designed an adaptive controller based on Lyapunov stability theory and the LaSalle invariant set theorem to control two chaotic PMSM systems with time-delay synchronization [18]. The error system of the two motors is asymptotically zero under the action of the controller, that is, the control method could realize the time-delay synchronization of the PMSM chaotic system. The numerical simulation results showed that the control method is correct and effective, and the time-delay coefficient greatly influences the performance of the system and is sensitive to changes in system model parameters. Xie et al. proposed a fuzzy adaptive synchronization control method for a chaotic PMSM system with unknown parameters [19]. The PMSM system parameters were assumed to be unknown, and the PMSM chaotic model and its response system model were expressed as a $T-S$ fuzzy model. Lyapunov stability theory and the adaptive control method were emplyed to design the response system, and the adaptive control law was derived to estimate the driving system parameters. The fuzzy controller of the response system was designed to synchronize the PMSM system and its response system, and the synchronization error dynamic was asymptotically stable. The simulation results verified the effectiveness of the proposed method, and although fuzzy control showed a robust performance, the structure of the proposed method is complex, and the determination of fuzzy rules depends on experience to a certain extent and is thus difficult to achieve. Wang et al. proposed a fuzzy adaptive synchronization control method for the chaotic motion of a PMSM with disturbance, analyzed the attractor and Lyapunov exponent spectrum of the chaotic motion of the PMSM, made the fuzzy control rules meet the Lyapunov stability conditions of the system, and designed a fuzzy adaptive controller to control the chaotic system with disturbance [20]. The simulation results showed that the proposed method could realize the synchronization control of the chaotic motion of PMSMs and has a good control effect. Although the proposed method effectively combines fuzzy control and adaptive control, the structure of the controller is complex, and the fuzzy rules are difficult to determine.

Convergence is an important performance index of any control system. In the aforementioned methods, the closedloop systems are applicable to exponential forms. Thus, these methods cannot guarantee that the system converges to the equilibrium point in a limited time. Finite-time stability control is a time-optimal control method with strong robustness and capability of guaranteeing the fast convergence to the equilibrium point [21]. Therefore, finitetime stability control in control systems has attracted the attention of scholars [22-26]. Pilloni et al. applied the finitetime stability control method to control the voltage and frequency of a microgrid on the basis of an islanding inverter, proposed an accurate finite-time recovery method, and verified the proposed method's effectiveness through simulation [22]. Tang et al. adopted the finite-time stability control method to the chaos synchronization of a PMSM with a nonuniform air gap [23]. The method has a strong robustness to changes in system parameters, but it does not consider the influence of such changes on system load disturbance. Wei et al. used the finite-time stability control method to the chaos suppression of PMSM [24]. When parameters change, the method still achieves high control performance, but its restrain of load disturbance is poor and even causes system instability. Aghababa et al. adopted finite-time stability control to realize the control and synchronization of a fractional chaotic system [25]. Wang et al. proposed the finite-time stability control of a unified chaotic system, but the stability time is long and needs further improvement [26]. The influence of load disturbance on dynamic system performance is not considered in the aforementioned study.

The studies previously described were mainly aimed at investigating the chaos synchronization control of PMSM and the control of system convergence. By contrast, studies on the dynamic performance of PMSM systems due to its load disturbance factor are rare. Equally inadequate is the study work related to the limited time chaos synchronization control of PMSM with a nonuniform air gap. Herein, sliding mode theory and finite time stability theory are employed to design chaos synchronization controllers, which meet system requirements from the time-optimal point of view. The load of the driving system is estimated in real time by a sliding mode observer. Finite-time stability theory is employed to realize the finite-time chaos synchronization of the system. The control method is further improved by introducing the terminal attractor proportion factor to improve the quick response of the system.

The remainder of this study is organized as follows. Section 3 designs the finite-time synchronization controller of the PMSM chaotic system based on sliding observer. Section 4 compares the three methods of this work with those in reference [3] and [26] through numerical simulation experiments to verify the effectiveness and superiority of the proposed method. Section 5 summarizes the conclusions.

\section{Methodology}

\subsection{Theoretical basis}

Definition For a dynamic system, there is $s X=f(X)$. If a certain time $T>0$ exists ( $T$ may be related to the selection of the initial state), then the two following conditions are both true: $\lim _{t \rightarrow T}\|X(t)\|=0$. If $t \geq T$ and $\|X(t)\|=0$ is constant, then the system is stable in finite time. Here, $X \in R^{n}$ is the n-dimensional state variable, $s$ represents the differential operator, and $f(x)$ is a smooth nonlinear function.

Lemma 1 [26] If continuous and positive definite function $V(t)$ exist, constant real numbers $m>0$ and $0<\xi<1$ meet the following conditions:

$$
s V(t) \leq-m V^{\xi}(t), \forall t \geq t_{0}, V\left(t_{0}\right) \geq 0
$$

Then, for any initial time $t_{0}$, the following inequality holds:

$$
V^{1-\xi}(t) \leq V^{1-\xi}\left(t_{0}\right)-m(1-\xi)\left(t-t_{0}\right), \forall t \geq t_{1}, V(t) \equiv 0
$$

where $t_{1}=t_{0}+\frac{V^{1-\xi}}{m(1-\xi)}$, that is, the time required for system stability. 
The proof of the theorem can be found in reference [26], which is not proven here. Only a simple analysis of the theorem is made. The expression of $t_{1}$ shows that when parameters $t_{0}$ and $\xi$ of the system are fixed, the stability time of the system becomes inversely proportional to parameter $p$, and a proper increase of $m$ can effectively shorten stability time. Thus, the controller of reference [26] is improved.

Lemma 2 [23] For nonzero positive real numbers $a, b$, and $c$, in which $c \in(0,1)$, the following inequality holds:

$$
a^{c}+b^{c} \geq(a+b)^{c}
$$

\subsection{Chaotic model of PMSM with nonuniform air gap}

The dimensionless mathematical model of a PMSM after transformation is as follows [3]:

$$
\left\{\begin{array}{l}
\tau_{1} s x_{1}=-x_{2} x_{3}-x_{1}+v_{d} \\
\tau_{2} s x_{2}=-x_{2}-x_{1} x_{3}-x_{3}+v_{q} \\
\tau_{3} s x_{3}=a x_{1} x_{2}+b x_{2}-c x_{3}-T_{L}
\end{array}\right.
$$

where $\left(x_{1}, x_{2}, x_{3}\right)=\left(i_{d}, i_{q}, w\right), v_{d}$ and $v_{q}$ are the voltages of the $d$-axis after transformation, $i_{d}$ and $i_{q}$ are the current and $q$-axis after transformation, respectively. $w$ and $T_{L}$ are the speed and equivalent load after transformation, respectively. $a, b$, and $c$ are the motor parameters. $s=d / d t$ are differential operators [3].

When $v_{d}=-12.70, v_{q}=2.34, T_{L}=0.525, \tau_{1}=7.125$, $\tau_{2}=6.45, \tau_{3}=1, a=1.516$, and $b=16, c$ is the bifurcation parameter affected by stator resistance and magnetic chain. The bifurcation diagram of the system is shown in Fig. 1. When $c=1.8$, the typical chaotic attractor can be made, as shown in Fig. 2. Only the phase diagram of $x_{1}$ is provided. The simulation is based on the MATLAB platform. The fourth-order Runge-Kutta method is used, the sampling time $T_{s}$ is $0.01 \mathrm{~s}$, and the initial state is $\left(i_{d 0}, i_{q 0}, w_{0}\right)=(1,1,1)$. In Fig. 1 and $2, i_{d}, i_{q}$, and $w$ are expressed as unit values.

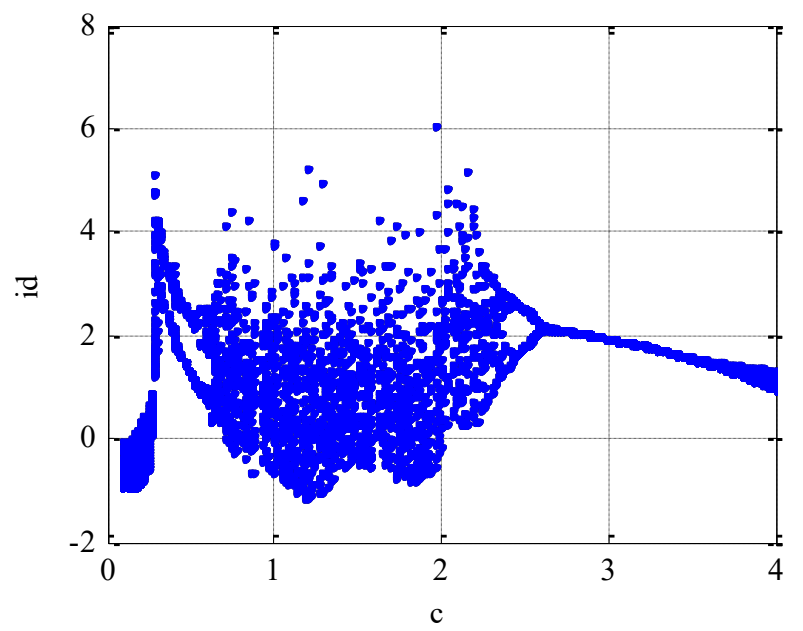

Fig. 1. Graphical illustration of the particle representation scheme

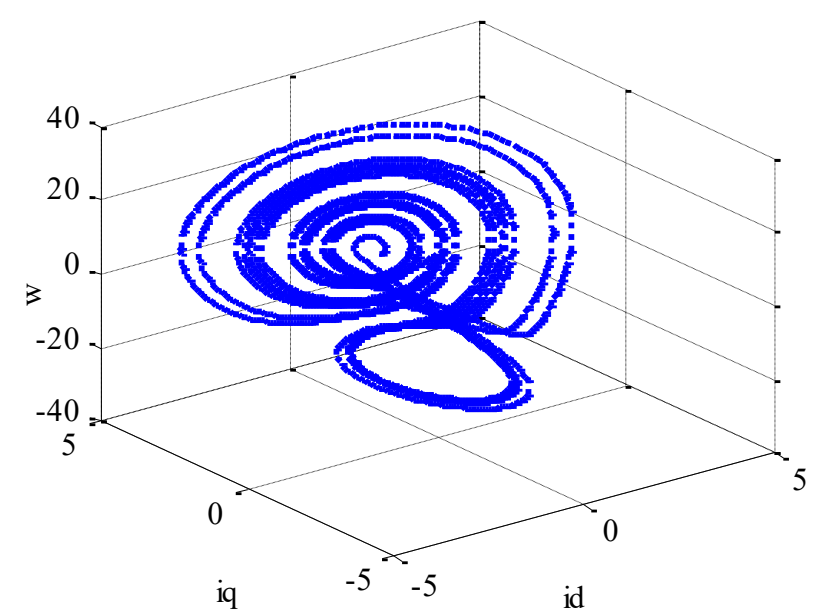

Fig. 2. PMSM chaotic attractor

\subsection{Design of sliding mode observer}

A load sliding mode observer with speed and load as the observation object is employed to realize the online estimation of the equivalent load. According to the actual situation, the frequency of the general controller is high. Thus, the load torque can be considered fixed in the control cycle, that is, $s T_{L}=0$. The resulting state equation and speed can be expressed as follows:

$\left\{\begin{array}{l}\tau_{3} s x_{3}=a x_{1} x_{2}+b x_{2}-c x_{3}-T_{L} \\ s T_{L}=0\end{array}\right.$

Then, the sliding mode observer constructed by Eq. (5) is expressed as follows:

$\left\{\begin{array}{l}\hat{\tau_{3} s x_{3}}=a x_{1} x_{2}+b x_{2}-c \hat{x_{3}}-\hat{T_{L}}+Q \\ \hat{s} \hat{T}_{L}=\gamma Q\end{array}\right.$

where $x_{3}$ and $\hat{T}_{L}$ are the speed and load estimates, respectively. $Q=\mu \operatorname{sign}(\sigma)$ and $\sigma=e_{w}=x_{3}-x_{3}$ are the sliding surfaces, $e_{w}$ is the speed estimation error, $\mu=-\varepsilon\left|e_{T}\right|$ is the sliding mode gain, $\varepsilon \geq 1$ is the adaptive gain, $e_{T}$ is the speed estimation error, and $\gamma$ is the feedback gain.

Theorem 1 The sliding mode observer shown in Eq. (6) is used for Eq. (5), and the system state error approaches zero exponentially.

The error equation of the sliding mode observer can be obtained by substituting Eq. (6) into Eq. (5) as follows:

$\left\{\begin{array}{l}\tau_{3} s e_{w}=-c e_{w}-e_{T}+Q \\ s e_{T}=\gamma Q\end{array}\right.$

The Lyapunov function is taken as $V_{0}=\frac{1}{2} \tau_{3} \sigma^{2}$ to derive the following equation:

$s V_{0}=\sigma \tau_{3} s \sigma=e_{w} \tau_{3} s e_{w}=e_{w}\left(-c e_{w}-e_{T}+\mu \operatorname{sign}\left(e_{w}\right)\right)$ 
$=-c e_{w}^{2}-\left(e_{w} e_{T}-\mu\left|e_{w}\right|\right) \leq-c e_{w}^{2} \leq 0$

Eq. (8) shows that the speed estimation error gradually approaches zero in exponential form.

When the sliding mode observer enters the sliding mode, it satisfies the following requirements $\sigma=s \sigma=0$, that is, $e_{w}=s e_{w}=0$, which can be obtained by substituting it into Eq. (7): $s e_{T}=\gamma e_{T}$ and $\gamma>0$. Therefore, the observation error approaches zero exponentially, $\gamma$ determines its approach speed, and the theorem is proven according to system stability theory.

The existence of the sign function in the sliding mode observer inevitably causes the chattering of the system. Thus, using the hyperbolic tangent function replaces the sign function, that is, $Q=\mu \tanh (\sigma)$, to further improve system performance.

\subsection{Design of finite-time synchronous controller}

The driving system is set as Eq. (4), and the response system is as follows:

$\left\{\begin{array}{l}\tau_{1} s y_{1}=-y_{2} y_{3}-y_{1}+v_{d}+u_{1} \\ \tau_{2} s y_{2}=-y_{2}-y_{1} y_{3}-y_{3}+v_{q}+u_{2} \\ \tau_{3} s y_{3}=a y_{1} y_{2}+b y_{2}-c y-\hat{T}_{L}+u_{3}\end{array}\right.$

Let $e_{i}=y_{i}-x_{i}$ and $i=1,2,3$. Then, the error system can be expressed as follows:

$$
\left\{\begin{array}{l}
\tau_{1} s e_{1}=e_{2} e_{3}+e_{2} x_{3}+e_{3} x_{2}-e_{1}+u_{1} \\
\tau_{2} s e_{2}=-e_{2}-e_{1} e_{3}-e_{1} x_{3}-e_{3} x_{1}-e_{3}+u_{2} \\
\tau_{3} s e_{3}=a\left(e_{1} e_{2}+e_{1} x_{2}+e_{2} x_{1}\right)+b e_{2}-c e_{3}-e_{T}+u_{3}
\end{array}\right.
$$

In Eq. (10), the controller is designed as follows on the basis of active control and finite-time stability theory.

Theorem 2 The following forms of controller are adopted for uncertain system Eq. (10):

$$
\left\{\begin{array}{l}
u_{1}=-e_{2} e_{3}-e_{2} x_{3}-e_{3} x_{2}-k_{1} e_{1}^{\alpha} \\
u_{2}=e_{3} x_{1}+e_{3}-k_{2} e_{2}^{\alpha} \\
u_{3}=-a e_{2} x_{1}-b e_{2}+e_{T}-k_{3} x^{\alpha}
\end{array}\right.
$$

where $k_{1}, k_{2}$, and $k_{3}$ are the terminal attractor weight coefficients and are all positive real numbers, $k_{1}=k_{2}=k_{3}=k$ are taken as a simplified calculation. $\alpha=p / q$, where $0<p<q . p$ and $q$ are all odd numbers, and the state error of Eq. (10) tends to be zero in finite time.

The following expressions can be obtained by substituting the controller $u_{1}$ into the first equation in Eq. (10):

$$
\tau_{1} s e_{1}=-e_{1}-k_{1} e_{1}^{\alpha}
$$

where Lyapunov function $V_{1}=0.5 \tau_{1} e_{1}^{2}$. The derivative along the path of Eq. (10) can be expressed as follows: $s V_{1}=e_{1} \tau_{1} s e_{1}=e_{1}\left(-e_{1}-k_{1} e_{1}^{\alpha}\right)=-e_{1}^{2}-k_{1} e_{1}^{\alpha+1} \leq-k_{1} e_{1}^{\alpha+1}$

$=-k_{1}\left(0.5 \tau_{1}\right)^{-0.5(\alpha+1)}\left(0.5 \tau_{1} e_{1}^{2}\right)^{0.5(\alpha+1)}=-m_{1} V_{1}^{\xi}$

where $m_{1}=k_{1}\left(0.5 \tau_{1}\right)^{-0.5(\alpha+1)} \quad$ and $\quad \xi=0.5(\alpha+1)$. If $0<\alpha<1$, then $0<\xi<1$, and $m>0$. Thus, system error $e_{1}$ approaches zero in finite time $t_{1}=e_{1}(0) /\left(k_{1}(1-\alpha)\right)$ according to Lemma 1.

By substituting $e_{1}=0, u_{2}$, and $u_{3}$ into Eq. (10), the following equations can be obtained:

$\left\{\begin{array}{l}\tau_{2} s e_{2}=-e_{2}-k_{2} e_{2}^{\alpha} \\ \tau_{3} s e_{3}=-c e_{3}-k_{3} e_{3}^{\alpha}\end{array}\right.$

where Lyapunov function $V_{1}=0.5\left(\tau_{2} e_{2}^{2}+\tau_{3} e_{3}^{2}\right)$. The derivative along the path of Eq. (14) can be expressed as follows:

$$
\begin{aligned}
s V_{2}=e_{2}\left(-e_{2}-\right. & \left.k_{2} e_{2}^{\alpha}\right)+e_{3}\left(-c e_{3}-k_{3} e_{3}^{\alpha}\right) \\
= & -e_{2}^{2}-e_{3}^{2}-k_{2} e_{2}^{\alpha+1}-k_{3} e_{3}^{\alpha+1} \\
\leq & k_{2} e_{2}^{\alpha+1}-k_{3} e_{3}^{\alpha+1} \\
= & -k_{2}\left(0.5 \tau_{2}\right)^{-0.5(\alpha+1)}\left(0.5 \tau_{2} e_{2}^{2}\right)^{0.5(\alpha+1)} \\
& -k_{3}\left(0.5 \tau_{3}\right)^{-0.5(\alpha+1)}\left(0.5 \tau_{3} e_{3}^{2}\right)^{0.5(\alpha+1)} \\
& \leq-m_{2} V_{2}^{\xi}
\end{aligned}
$$

where $m_{1}=\min \left(k_{2}\left(0.5 \tau_{2}\right)^{-0.5(\alpha+1)}\right.$ and $\left.k_{3}\left(0.5 \tau_{3}\right)^{-0.5(\alpha+1)}\right)$. If $0<\alpha<1,0<\xi<1$, and $m>0$, then system error $e_{1}$ and $e_{2}$ approaches zero in finite time $t_{2}$ according to Lemma 1 .

In summary, when $t>t_{2}$, the state error of the system Eq. (10) under the action of the controller Eq. (11) approaches zero in a limited time. Hence, the drive system Eq. (4) and response system Eq. (9) achieve synchronization in a limited time.

\section{Result Analysis and Discussion}

This part is mainly divided into two aspects to verify the simulation: one is to verify the design effect of the sliding mode observer, and the other is to illustrate the advantages of the proposed method by comparing it with the adaptive synchronization method (reference [3]) and the traditional finite-time method (reference [26]). The fourth-order Runge-Kutta method is used in the simulation, the sampling $\operatorname{tim} T_{s}$ is $0.01 s$, the initial conditions $\left(x_{1}, x_{2}, x_{3}, y_{1}, y_{2}, y_{3}\right)$ are $(1,1,1,5,5,5), c$ is 1.8 , and the other parameters are the same as those in Section 3.

\subsection{Observer performance verification}

A sudden change load is applied to the system to verify the performance of the observer. When $t \leq 50 \mathrm{~s}$, the sudden change $T_{L}$ is 0.525 . When $t>50 \mathrm{~s}$, the sudden change $T_{L}$ is 10. The simulation results are shown in Fig. 3. 
Chuansheng Tang, Jie Yang, Qiang Zhao, Xuandong Wu and Tao Li /

Journal of Engineering Science and Technology Review 13 (2) (2020) 63 - 71

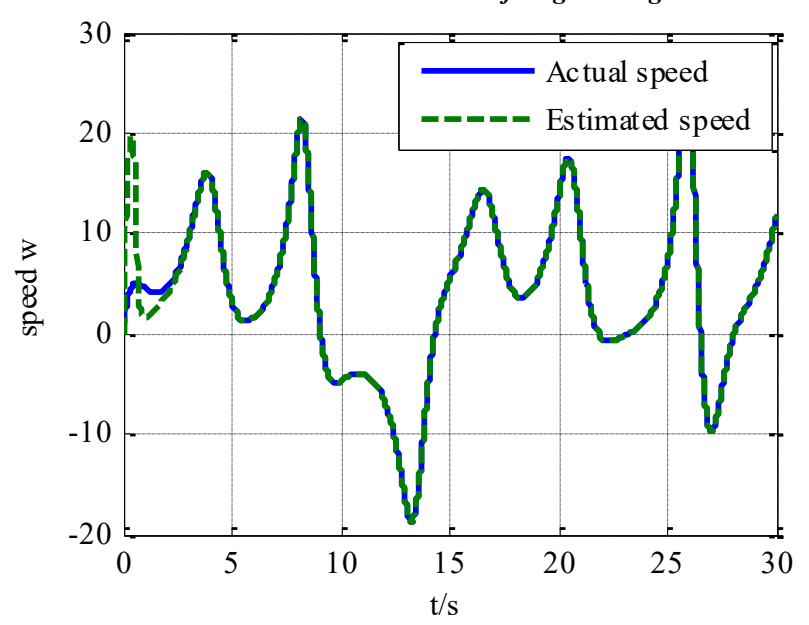

(a) Speed synchronous response curve

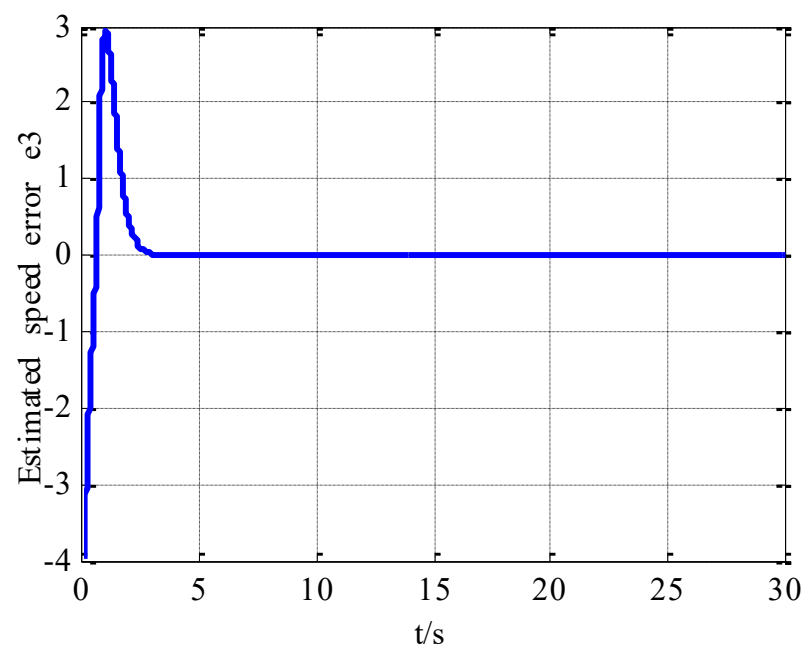

(b) Speed synchronization error response curve

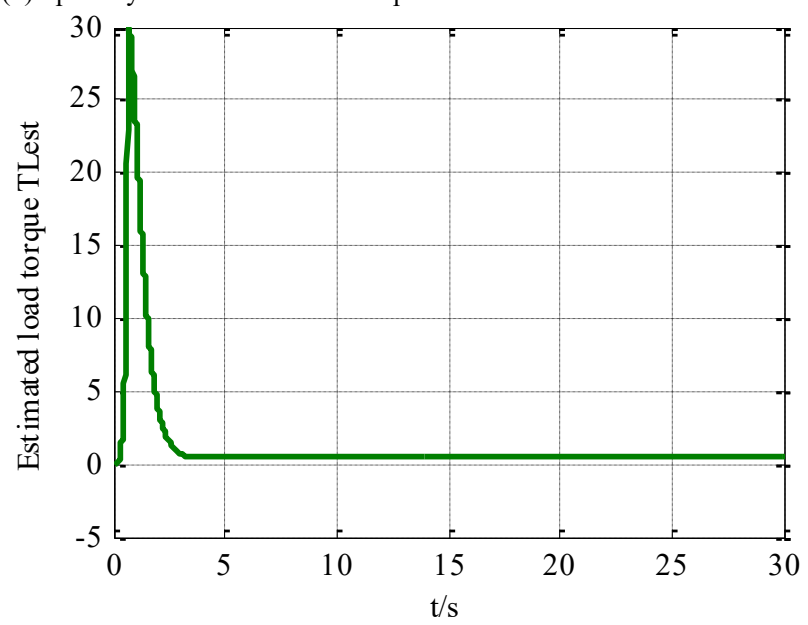

(c) Online load estimation

Fig. 3. Response curve of system with variable loads

Fig. 3(a) and (b) show that when the system changes from 0.525 to 10 in $15 s$, the response system can track the system well in a short time with strong robust performance. Fig. 3(c) shows that the designed sliding mode observer can accurately and rapidly track the sudden load, has no chattering in the steady state, and possesses good response and steady-state performance.

\subsection{Controller performance verification}

4.2.1 System performance comparison without parameter uncertainty

The controller parameters are $k_{1}=k_{2}=k_{3}=k=10$ and $\alpha=7 / 9$. The parameters of finite-time synchronous control (reference [26]) are $k=0.9$ and $\alpha=7 / 9$. The simulation results are shown in Fig. 4.
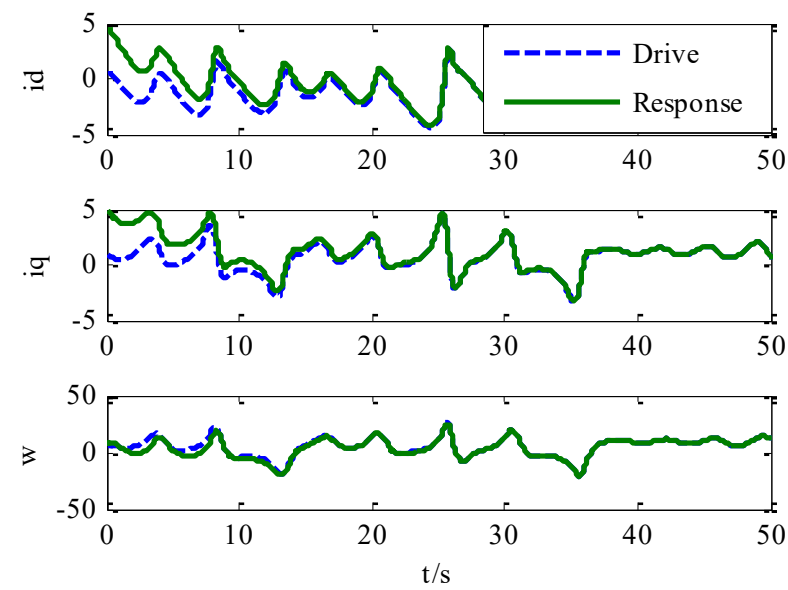

(a) State response curve of adaptive synchronization system
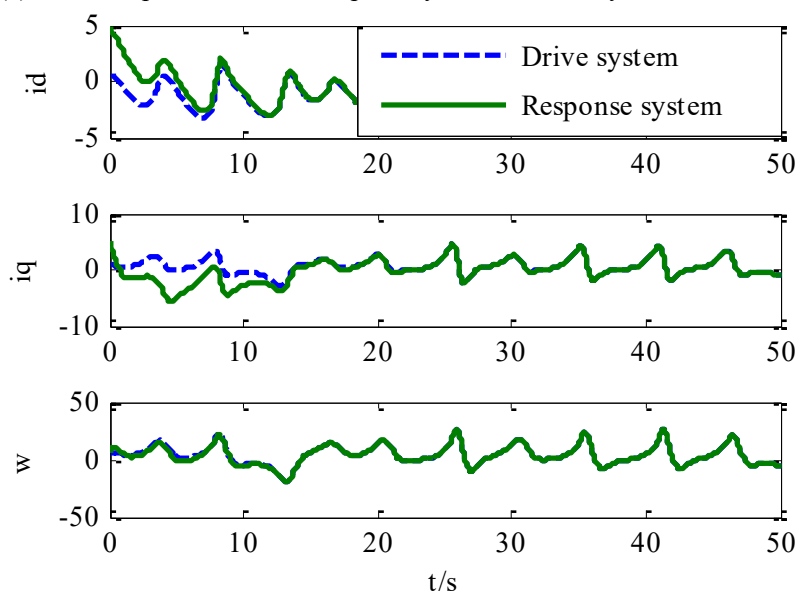

(b) State response curve of finite -time synchronous system
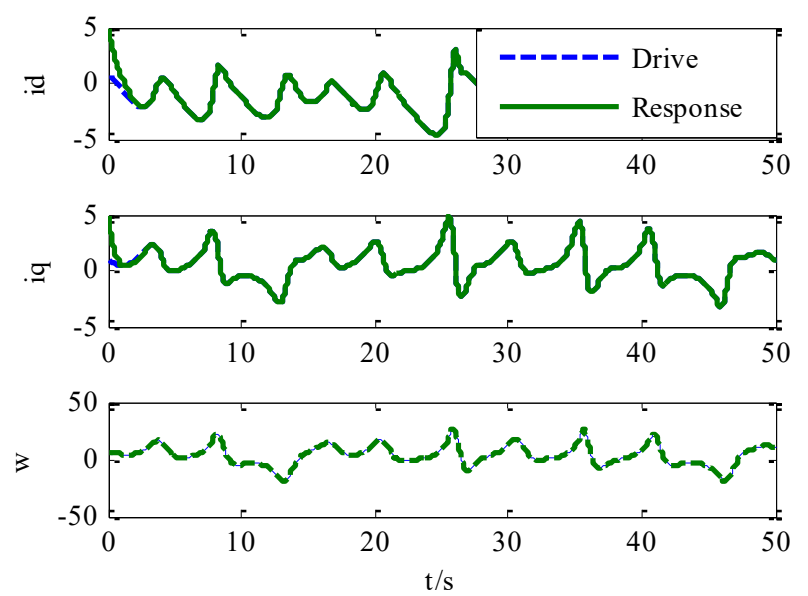

(c) System state response curve of the proposed method

Fig. 4. Response curvecurves of chaotic synchronization state of PMSM with nonuniform air gap

Fig. 4 shows that three control methods can realize the synchronization of the response system and the drive system, but the proposed method can realize the complete 
Chuansheng Tang, Jie Yang, Qiang Zhao, Xuandong Wu and Tao Li /

Journal of Engineering Science and Technology Review 13 (2) (2020) 63 - 71

synchronization of the response system and the drive system within $2 s$ under the same control parameters. Adopting the adaptive synchronous control method (reference [3]) takes $20 s$, but realizing the synchronization between the response system and the drive system by using the traditional finitetime synchronization control method takes $13 \mathrm{~s}$ (reference [26]). Therefore, the proposed method has faster response and can better realize system synchronization quickly compared with adaptive synchronization and finite-time synchronization.

\subsubsection{Performance comparison in case of parameter} uncertainty

To further verify the robust performance of the system, we assume that parameter $c$ of the system has $30 \%$ perturbation, which can be realized by rand() in MATLAB. The comparison between the adaptive synchronization method and the proposed method and the synchronization error response curve of each state variable are shown in Fig. 5, where the current error unit is $A$ and the speed unit is $m / s$.

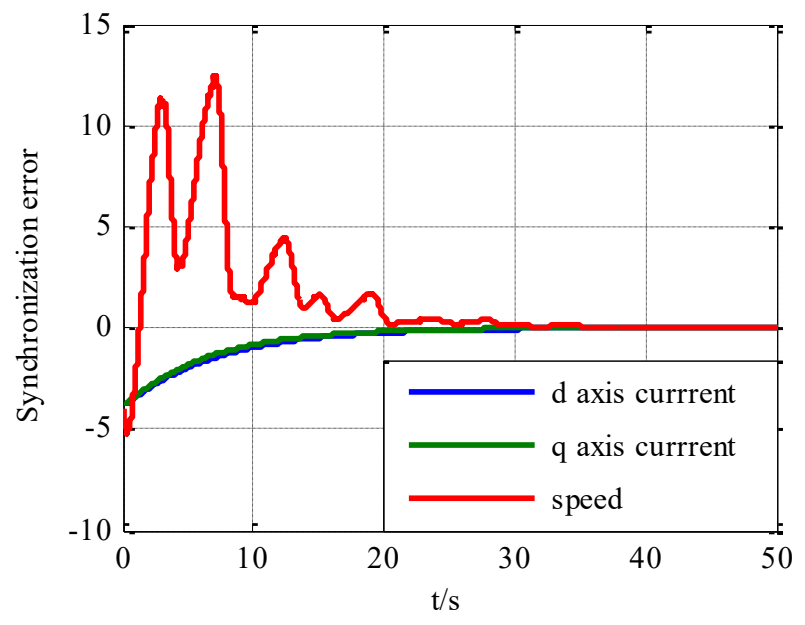

(a) Adaptive synchronization error

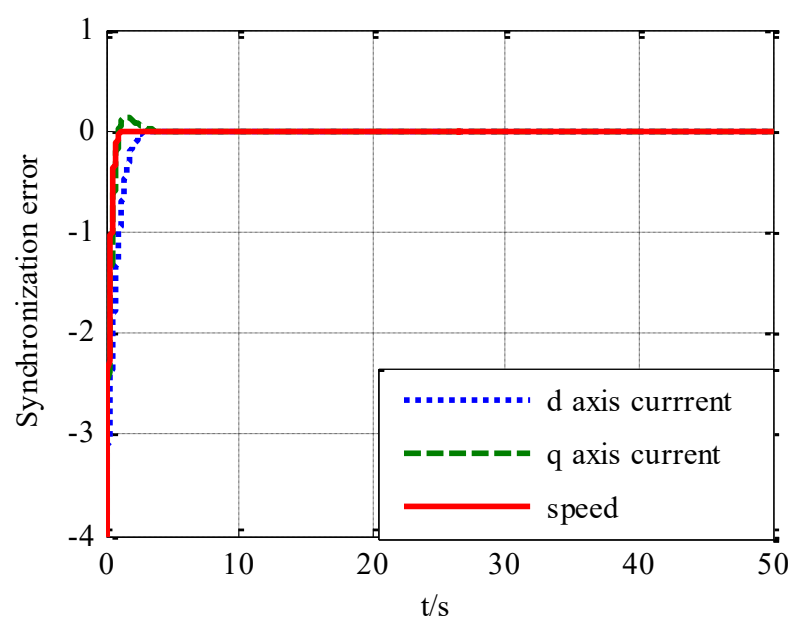

(b) The proposed method synchronization error

Fig. 5. Synchronous error response curve of system with parameter uncertainty

Fig. 5 shows that parameter $c$ of the system has $30 \%$ perturbation. The following conclusions can be drawn: (1) The adaptive synchronization method (reference [3]) introduces the adaptive mechanism, which can well realize the compensation of the system. However, realizing the full synchronization of the system takes about $32 s$. The proposed method can quickly realize the full synchronization of the system in approximately $3.5 \mathrm{~s}$, and the change of parameters has strong robustness. (2) The velocity error in the initial stage fluctuates greatly in reference [3], the fluctuation value reaches $13 \mathrm{~m} / \mathrm{s}$, whereas only the q-axis current fluctuates slightly in the proposed method with a fluctuation value of approximately $0.2 \mathrm{~A}$. Therefore, the proposed method has evident advantages over the method in reference [3].

\subsubsection{Performance comparison between uncertain} parameter and load disturbances

To further verify the robust performance of the system, we assume that parameter $c$ of the system has $30 \%$ perturbation and that the load disturbance changes from $0.525 \mathrm{~N}$ to $10 \mathrm{~N}$ in $15 \mathrm{~s}$. This study compares the adaptive synchronization method [3] with the proposed method, as shown in Fig. 6, where the current error unit in is $A$ and the speed single bit is $m / s$.

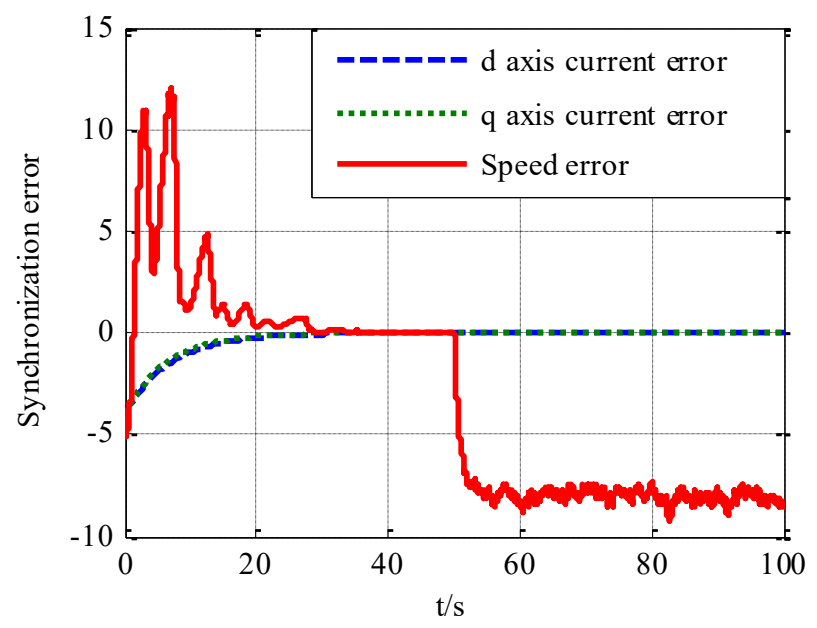

(a) Adaptive synchronization

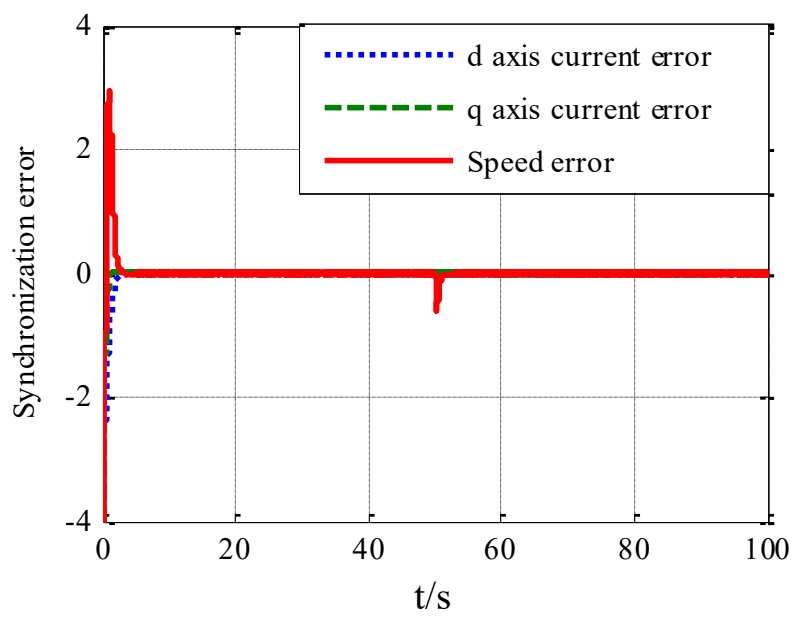

(b) The proposed method

Fig. 6. Response curves of system synchronization error with load disturbance and parameter 
Chuansheng Tang, Jie Yang, Qiang Zhao, Xuandong Wu and Tao Li/

Journal of Engineering Science and Technology Review 13 (2) (2020) 63 - 71

The following conclusions can be drawn from Fig. 6: (1) In the initial stage, the uncertainty of parameters plays an important role, and the conclusion is the same as that in Section 4.2.2. (2) When the load is suddenly changed $(t>=50 \mathrm{~s})$, the proposed method realizes real-time observation and compensation of load disturbance through the sliding mode observer. When $t$ is $50 \mathrm{~s}$, the speed and current of the system fluctuates slightly and quickly recover to state synchronization with the driving system. (3) The self-adaptive synchronization method (reference [3]) does not adopt external interference feedback. The load acts on the speed state equation, which makes the response system deviate from the state of the drive system. Thus, the speed synchronization control cannot be realized. Therefore, relative to the adaptive synchronization method, the proposed method possesses not only a good start-up and antisystem parameter time-varying performance but also a strong ability to suppress load disturbance.

\section{Conclusions}

The mathematical model of a PMSM with a nonuniform air gap was studied to overcome the influence of load disturbance and parameter uncertainty on the chaotic system of the PMSM with a nonuniform air gap. According to the strong coupling nonlinear characteristics, a sliding observer and a finite-time chaotic synchronization controller were designed and compared with adaptive synchronization control and finite-time synchronization control. The following conclusions could be drawn:

(1) In the presence of load disturbance, the designed sliding mode observer can effectively realize the online observation of loads. When the load suddenly changes, the designed observer can quickly and accurately track the load changes to facilitate real-time feedback to the controller for compensation.

(2) When the system parameters are invariable, the proposed method can realize the complete synchronization of the response system and drive system faster than the finite-time synchronization and adaptive control methods under the same control parameters.

(3) When the system has time-varying parameters, the dynamic tracking performance of the proposed method will be unchanged. However, the synchronization time of the two systems using the adaptive synchronization control method (reference [3]) is about 10 times that of the proposed method.

(4) When time-varying parameters and load disturbances are present, the proposed method can still quickly achieve the complete synchronization of the system state. However, the existence of load disturbances makes achieving accurate tracking synchronization of the system difficult without the adaptive synchronization of the load observation compensation.

Thus, an active finite-time synchronous control method based on a sliding observer is proposed by combining theoretical derivation with numerical simulation. The proposed method considers the influence of parameter changes and load disturbances on the chaos control and synchronization of PMSM, these conditions are close to the actual working conditions of motor chaotic systems. The results of this work offer a certain reference value for improving motor synchronous control performance. Given the lack of actual data of field motor parameter tests in motor modeling, future study could combine motor parameter test data with the proposed model and modifying it to achieve an accurate understanding of the nonlinear characteristics of complex motor chaotic system with strong coupling.

\section{Acknowledgements}

This work was supported by the Key Scientific Research Projects of Higher Education Institution of Henan Province (Grant No. 20B470003 and Grant No. 18B470007) and the Promotion Special Project of Scientific Research Program of Henan Province(Grant NO. 202102210298 and Grant No. 202102210084).

This is an Open Access article distributed under the terms of the Creative Commons Attribution License

\section{References}

1. Zhou, G. T., Wang, Z., Cheng, M., "Chaotic motor drives and its applications". Beijing: Science Press, China, 2009, pp.6-13.

2. Kuroe, Y., Hayashi, S., "Analysis of bifurcation in power electronic induction motor drive systems". In: Proceedings of the 20th international conference on power electronics specialists, Milwaukee, USA: IEEE, 1989, pp.923-930.

3. Zhang, X. H., Ding, S. G., "Adaptive chaos synchronization of nonuniform air gap permanent magnet synchronous motor". Control Theory and Application, 26(6), 2009, pp.661-664.

4. Lin, L. X., Huang, G. H., Peng, X. F., "Single input feedback global exponential stability control of PMSM chaotic system". Journal of Xiamen University (Natural Science Edition), 58(5), 2019, pp.748753.

5. Ge, Z. M., Cheng, J. W., Chen, Y. S., "Chaos anticontrol and synchronization of three time scales brushless DC motor system". Chaos, Solitons and Fractals, 22(5), 2004, pp.1165-1182.

6. Yin, J. S., Lei, T. F., Chen, H., et al., "Synchronous control of chaotic system of BLDCM based on Chua system". Journal of Jining University, 36 (6), 2015, pp.26-31.

7. Torres, F. J., Guerrero, G. V., García, C. D., et al., "Master-slave synchronization of robot manipulators driven by induction motors". IEEE Latin America Transactions, 14(9), 2016, pp.3986-3991.
8. Ranjbar, N. A., Kholerdi, H. A., "Chaotification and fuzzy PI control of three-phase induction machine using synchronization approach". Chaos Solitons and Fractals, 91, 2016, pp.443-451.

9. Kim, S. S., Han H. C., "Adaptive synchronization method for chaotic permanent magnet synchronous motor". Mathematics and Computers in Simulation, 101, 2014, pp.31-42.

10. Zhang, Z., Chau, K. T., Wang, Z., "Chaotic speed synchronization control of multiple induction motors using stator flux regulation". IEEE Transactions on Magnetics, 48(11), 2012, pp.4487-4490.

11. Liu, L., Cartes, D. A., "Synchronisation based adaptive parameter identification for permanent magnet synchronous motors". Institution of Engineering and Technology Control Theory and Applications, 1(4), 2007, pp.1015-1022.

12. Yang, X. H., Liu, X. P., Hu, L. L., et al., "Robust sliding mode variable structure chaos synchronization control of permanent magnet synchronous motor". Modular Machine Tool and Automation Manufacturing Technology, (8), 2012, pp.93-95.

13. Yu, Y., Mi, Z. Q., Liu, X. J., "Chaotic motion analysis and sliding mode control of doubly fed wind turbine". Journal of physics, 60 (7), 2011, pp.112-119. 
Chuansheng Tang, Jie Yang, Qiang Zhao, Xuandong Wu and Tao Li /

Journal of Engineering Science and Technology Review 13 (2) (2020) 63 - 71

14. Zaher, A. A., "A nonlinear controller design for permanent magnet motors using a synchronization-based technique inspired from the Lorenz system". Chaos, 2008, doi: 10.1063/1.2840779.

15. Vajiheh, V., Kheiri, H., Javidi M., "Chaotic dynamics and synchronization of fractional order PMSM system”. Sahand Communications in Mathematical Analysis, 2(2), 2015, pp.83-90.

16. Wang, X. Y., Zhang, H., "Backstepping-based lag synchronization of a complex permanent magnet synchronous motor system". Chinese Physics B (English version), 22(4), 2013, pp.558-562.

17. Yang, X. H., Liu, X. P., Liu, H.S., et al., "Synchronous control of chaotic motion based on the backstepping method of permanent magnet synchronous motor". Electrical Measurement and Instrumentation, 49 (12), 2012, pp.37-40.

18. Li, J.C., Wei, D. Z., Luo, X. S., Zhang, B., "Adaptive lagsynchronization of chaotic permanent magnet synchronous motor". Vibration and Shock, 33 (16), 2014, pp.105-108.

19. Xie, C. R., Zhang, R. Y., Wang, R. M., et al., "Fuzzy adaptive synchronous control of PMSM chaotic system with unknown parameters". Journal of Dynamics and Control, 15 (6), 2017, pp.537-543.

20. Wang, L., Li, Y. H., Zhu, X. H., et al. "Fuzzy adaptive synchronization of chaotic motion of PMSM with disturbance". Power System Protection and Control, 39 (11), 2011, pp.33-37, 43.
21. Hwang, C. L., Hung, J. Y., "Stratified adaptive finite-time tracking control for nonlinear uncertain generalized vehicle systems and its application". IEEE Transactions on Control Systems Technology, 27(3), 2019, pp.1308-1316.

22. Pilloni, A., Pisano, A., Usai, E., "Robust finite-time frequency and voltage restoration of inverter-based microgrids via sliding-mode cooperative control". IEEE Transactions on Industrial Electronics, 65(1), 2018, pp.907-917.

23. Tang, C. S., Dai, Y.H., Zhen, W.X., "Finite-time chaotic synchronization of permanent magnet synchronous motor with nonsmooth air-gap". Control Theory and Applications, 31(3), 2014, pp.404-408.

24. Wei, D. Q., Zhang, B., "Controlling chaos in permanent magnet synchronous motor based on finite-time stability theory". Chinese Physics B, 18(4), 2009, pp.1399-1403.

25. Aghababa, M. P., "Finite-time chaos control and synchronization of fractional-order nonautonomous chaotic (hyperchaotic) systems using fractional nonsingular terminal sliding mode technique". Nonlinear Dynamics, 69(1-2), 2012, pp.247-261.

26. Wang, H., Han Z. Z., Xie, Q, Y, Zhang, W., "Finite-time chaos synchronization of unified chaotic systems with uncertain parameters". Communications in Nonlinear Science and Numerical Simulation, 14(5), 2009, pp.2239-2247. 